\title{
Integration of Augmented Reality into College Yearbook
}

\author{
Faharol Zubir ${ }^{1, *}$, Ina Suryani ${ }^{1}$, and Norlizawati Ghazali ${ }^{2}$ \\ ${ }^{1}$ Centre for International Languages, Universiti Malaysia Perlis. \\ ${ }^{2}$ Academy of Language Studies, Universiti Teknologi MARA.
}

\begin{abstract}
Augmented reality (AR) has been used in many schools and colleges in developed countries. Apart from enhancing teaching to be more interactive, inventive and resourceful, the use of AR also has been reported to boost learning by fostering creativity and imagination, enhancing collaboration and team work, and making learning more engaging and stimulating. Despite the potentials of AR in enhancing school materials, this technology often has teachers and learners as the end users rather than developers. Given the benefits of AR in education, this study experimented on having the teachers and learners as developers in incorporating the use of AR to enhance the college yearbook. This paper describes the processes taken by the teachers and learners in integrating AR into a conventional reading material; specifically a college yearbook. In this study, an open source AR application is used and 32 markers are created. The method involves five major development processes which starts with planning, content, AR integration development, printing and finally AR integration construction. The 32 markers can be divided into a few sections which can be classified according to the types of content. At the end of this paper, the significance and challenges of this project are presented.
\end{abstract}

\section{Introduction}

College yearbook thus far, comes in hardback, paperback and lately in PDF formats. It is considered a conventional book since it comes with text and illustrations only. Often, publishing a yearbook has a lot of constraints particularly the on the finance and readership issues. Some colleges have stopped publishing the physical yearbook altogether due to the financial factor. One of the main problems is the high cost in making the yearbook interactive and attractive to the teenage readers. In order to attract the college students the yearbook needs to have many illustrations such as pictures and colorful pages of articles hence increase the printing cost further. Due to these issues, developing a conventional yearbook to students in the 21 st century can be a daunting task for any yearbook advisor.

Opportunely, the integration of AR can enhance the yearbook to be more interactive and promote better readership without escalating the printing cost. Moreover, the yearbook is often associated with momentous occasions and it is constructed collectively by teachers and students as such, presents potentials for AR experimentation. Such meaningful, fun medium ease the learning of the new technology which otherwise is daunting to both teachers and students. While being meaningful as it records important events, yearbook construction involves limited number of editorial members. This research involves a project that accommodates participations from students and teachers. AR enhances experience of the real world by the use of virtual content which is overlaid onto the reality. AR presents new features that can be mixed with our perception. The new features in the form of $2 \mathrm{D}, 3 \mathrm{D}$ objects, audio, video files, textual information alters the perceptions and can be incorporated into the users' perceptions of the real world [1].

Recognizing these potentials, this study attempts to develop a yearbook by enhancing its quality and sustainability through the use of AR. In the process of the development, this study also examines the development of the instructional design for $\mathrm{AR}$ integration in teaching and learning. This paper reports on the development process of the yearbook, the incorporation of AR elements into the yearbook enhancement and the roles played by teachers and learners in completing this new yearbook.

\section{Theory and Method}

This section has two parts whereby the first part discusses on the previous researches on AR in education and the second part describes the methods used to integrate AR elements into the yearbook. The second part also illustrates the processes undertaken by the teachers and learners in achieving the expected yearbook.

\subsection{Theory}

Augmented reality (AR) can be defined as a technology which overlays virtual objects or augmented components into the real world. These virtual objects then display to coexist in the same space as objects in the real world [2]. $\mathrm{AR}$ is currently a popular technology that is widely used in marketing and entertainment settings. In recent years, $\mathrm{AR}$ has become the focus of many researches $[3,4]$. The

* Corresponding author: faharol@unimap.edu.my 
fact that $\mathrm{AR}$ no longer requires expensive hardware and sophisticated equipment, such as head-mounted displays (HMD) contributes to its popularity. The technology now can be accessed through computers or mobile devices. The rapid development of free AR application contributes to its vast accessibility towards users. Therefore, using AR technology is not as challenging as it was in the past.

Today, in the developed countries, AR is often used in every level of schooling [5, 6]; however this technology is hardly ever utilized or employed by the academics and students in Malaysia. One of the emerging application of AR in education is in the form of augmented books. The potential of digitally augmented books usage appeals to researchers and educators. Augmented books provide a means for enhancing regular 2D books with interactive visualizations, animations, 3D graphics, audios and videos. The purpose of integrating such features is to promote engaging user experiences and to enhance the reading experience.

To date, on the global scale, the number of book readers has declined rapidly. The development and production of conventional reading materials often limit readers' involvement particularly in the inclusion of additional reading materials. Therefore, the integration of multimedia using AR in reading materials, particularly yearbook, appeals to the readers' interest and may sustain the readership. Several research such have been done in Malaysia in terms of enhancing conventional reading material using AR integration such as $[7,8$, and 9$]$.

Despite the growing interest amongst publishers to incorporate $\mathrm{AR}$ into books, teachers and students are seldom involved or participated in the development of such book. Moreover, the technology is relatively new; consequently, the teachers and students only transpire as end users. Not wanting to miss out on the advantages, benefits and ease of incorporating AR in instructional materials, this study looks at the processes of transferring AR technology into teaching and learning materials. The yearbook is chosen to be the medium of transfer as the production of a yearbook is most likely to be a memorable project as it is associated with momentous occasions and constructed collectively. Such meaningful and fun medium ease the learning of the new AR technology which otherwise can appear daunting and overwhelming for the teachers and students. The knowledge can later be applied into other educational applications such as enhancements for textbooks, modules and students' projects.

\subsection{Methodology}

This study is conducted using a yearbook project which runs for 11 weeks. This yearbook project is a collaborative work by the International English Course
(IEC) students of UniMAP and is supervised by the language teachers. This section explains briefly about the IEC background and the previous conventional yearbook. The development of the AR integration particularly on the process is described at the end of this section.

The International English Course aims to prepare international students for UniMAP undergraduate programs and to improve the international students' level of English so that they are able to gauge the learning at the undergraduate programs in UniMAP which are conducted in English. The students are taught according to the four components of the English language skills which are Reading, Writing, Listening and Speaking. The learners are grouped into three levels according to their proficiency.

Previously, the yearbook is developed by IEC learners from the Intermediate group only with the other levels as the content contributors. It is a project aimed to showcase students' ability to write in order to reflect on their language lessons. Students are given the freedom to suggest the article they want to write about and the instructors guide them through the process of writing the articles. There are now 3 volumes of the yearbook. Volume 1 consists of essays written by students from the Upper-intermediate class and focuses on academic topics. Volume 2 on the other hand has a mixture of contributors from Intermediate, Pre-intermediate and Elementary class. The focus of the written materials is more towards short paragraphs on experiences and simple topics. Both volumes do not stress on the format of the write up since the main purpose of having the yearbook is to promote students to write.

In this project, the yearbook which is integrated with AR is developed by the October 2016 Intake. Unlike the previous volume, Volume 3 is a collection of academic essays written using a process-based approach. These essays are enhanced through the integration of AR in the yearbook. The yearbook consists of 46 coloured pages and is printed in A4 size. The yearbook consists of six sections namely the front matters, academic essays, additional articles, interviews, IEC students' profile and credit page.

The front matters include table of content, note from IEC Program Chairperson and the Head Editor, information on the team of authors and instruction on utilizing $\mathrm{AR}$ integration in the yearbook. The front matters consist of 6 pages (page 1-6). The next section is the academic essay section consisting of 22 pages (page 8-29). This section displays a collection of academic essays written by the students. The topics of the essays are Tourism in Malaysia, Vacation, The Importance of Sports, How to Achieve Your Goals, The Amazing Ancient Egypt, Essential Skills for University Students and The Benefits of Internet.

The additional articles section consists of two articles, Our Life in IEC and Augmented Reality \& Aurasma covering 3 pages (33-35). The next section is 
the Interview section which consists of 2 pages (37-38) about two IEC instructors. The last section is the IEC students section which displays a picture of each student in IEC October 2016 intake together with their names, country, offered undergraduate program and life motto. This consists of 7 pages (39-45). The last page consists of the credit page listing the name of the project advisors, head editor and compiler, team of writers and picture resources.

The AR integrated sections are the academic essays, additional articles and IEC students' profile. The selection of these sections is based on the purpose to showcase students' work. This is apparent in the academic essay and additional article section whereby the essays and articles are AR integrated with video overlays to provide additional information on each essay and article. The IEC students' profile on the other hand was chosen to maximize students' involvement in the yearbook. Each student records a 10 second video which are overlaid on their profile picture in the section. This ensures that more students can participate in the construction of the yearbook compared to the conventional way where only limited number of students can participate.

The process of integrating AR into the yearbook consists of 5 steps. The process starts with Step 1 which is the Planning step. In this step, the instructor briefs the students about the project that they are going to embark. The next process is to introduce students to AR and the chosen application which is Aurasma. This is then followed by assigning the person in charged for each section of the yearbook and also for the integration of $\mathrm{AR}$ in it.

Step 2 involves the process in developing the content of the essay which is the academic essays. Each student is assigned to write an academic essay for the yearbook. The first process in Step 2 is that each student is instructed to choose a topic of their interest and consult the instructor regarding their selection. Once both parties have agreed on the chosen topics, students can proceed to gather suitable materials needed to write the essay. The next process is to brainstorm for ideas and then construct an essay outline. Students are then required to submit the draft of their essay to the instructor. The instructor checks the drafts and suggests improvements for the drafts. The process is repeated until the drafts meet the required standards aimed by the International English Course. Once both parties are satisfied with the draft, the students proceed to submit the academic essay.

Step 3 includes the AR integration development process. The type, size and location of the markers are determined in this step. Next, the type, size, duration and resolution of the overlays are determined. Students are given the freedom to suggest and decide on these criteria for the markers and overlays after consulting the instructor. Step 4 involves the printing process of the yearbook. The formatting process is done and the yearbook is drafted. Once both parties, students and the instructor are satisfied with the draft, the printing process begins. The end product of this step is the hardcopy of the yearbook.

The next step is the AR integration construction. This step includes the process involved in integrating AR with the yearbook. Firstly, the selected open source AR application which is Aurasma is downloaded. Next, the technical editor registers a free Aurasma account and creates a channel for the yearbook. After that the technical editor tags the overlays to the markers available in the yearbook. Then the markers are tested to make sure that the overlays are retrievable. Once all the overlays for each marker works, the yearbook administrator, in this case the instructor, secures the tagging of the yearbook by controlling the access of the Aurasma account and channel for the yearbook. This is to ensure the safety of the content of the overlays triggered by the markers.
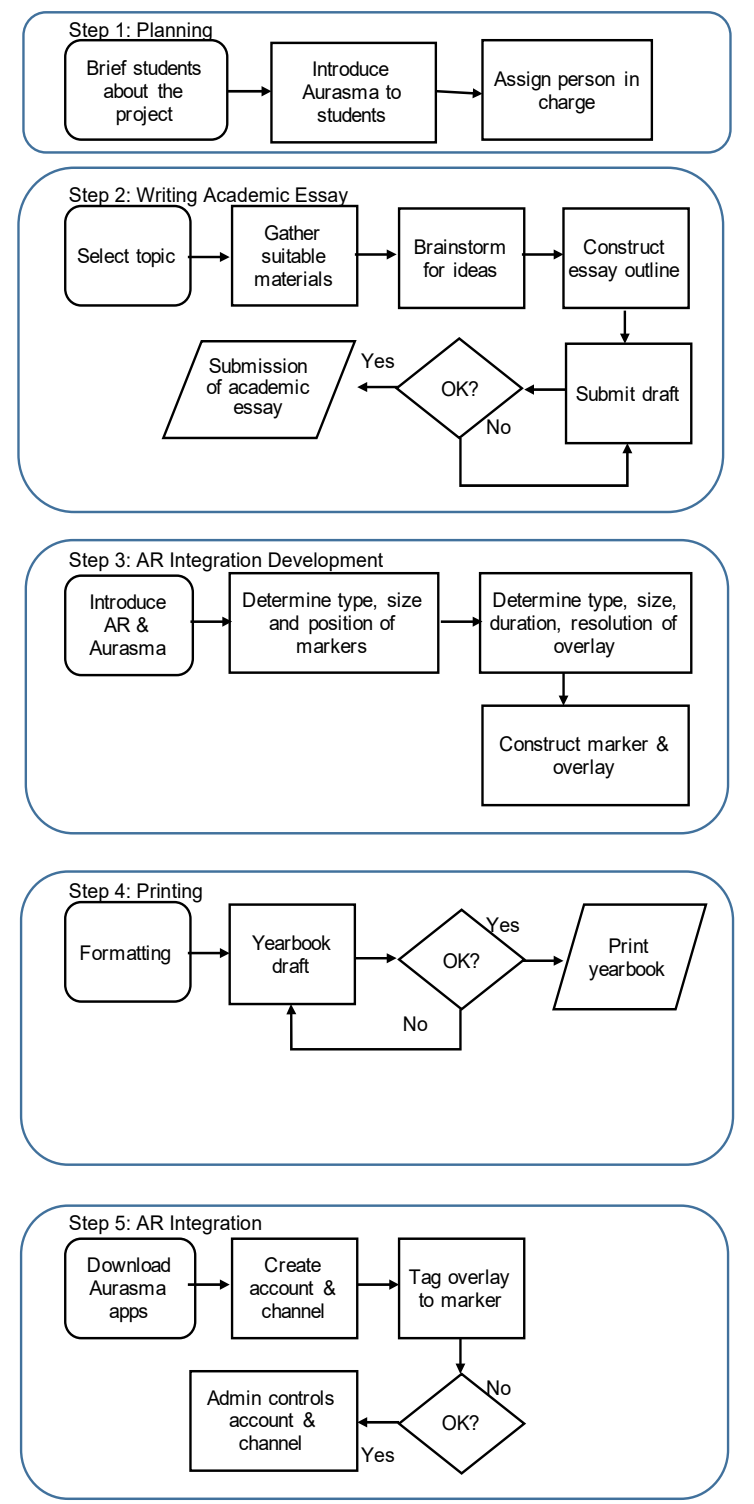

Fig. 1. The processes of AR Integration into yearbook 
A survey was done to evaluate if the AR integration enhance the readers' interest and to compare the users' experience when reading the book with and without AR integration. The survey was adopted and adapted from [10] and added with five items for publication rating.

The survey was conducted among 77 college students taking English language course as part of their program. The survey was conducted in two stages. In the first stage the subjects were given 15 minutes to read the book. Then, they were asked to answer the readership survey question. Next, the integration of AR was explained and the use of it is briefed to the subjects. They were then given another 15 minutes to re-read the book by using the AR integration function. After that, they were asked to answer the readership survey question again. This is to compare their rating on the content, design, quality, user friendliness and reader appeal aspects of the yearbook. A 5 point Likert scale (1. very poor, 2 . poor, 3 . average, 4 . good, and 5. excellent) were used in the readership survey questions.

\section{Result}

The outcomes of the project suggest three main considerations which are sections, markers and instrumentation. The first consideration on the section is related to the suitability of AR integration with the content. The yearbook consists of six sections namely the front matters, academic essays, additional articles, interviews, students' profile and credit page. AR is integrated throughout the yearbook except for two sections; front matters and interviews.

The second consideration is on the use of markers. Markers refer to the image in the yearbook that can be scanned in order to retrieve the overlays. Overlays on the other hand are materials; images, videos, links etc. that are projected on top of the marker once it is scanned. The markers need to be carefully planned and placed since the markers are visible in the reading material and once the printing process is completed, no further amendments can be done onto them. However, this is in contrast with the overlay which can be further enhanced or replaced even after the completion of the printing process. Markers utilize image recognition process and they work best if both pictures and texts are included in the same marker. The more complex and the more unique the markers are, the better they function. In this yearbook, the markers are combinations of images and texts. Each marker is bordered by a box with green background and an Aurasma logo. This is to ensure the visibility and uniformity of the markers for better recognition. The markers are relatively quite large, with some even covering a whole page. This is to ease users when they scan it. For the academic essays and additional articles, the markers are placed at the last page of each article. By doing so, readers are able to read the article without distractions from the markers. Since the overlays function as a summary for the articles, the articles need to be read first.
There are altogether 32 markers in the yearbook, which are put into three sections of the yearbook. Seven markers are included in the academic essay and additional articles section. Among the seven, four markers are triggered for a self-recorded video that are overlaid on top of the academic essay. These are the oral summary of the essays recorded by the respective authors of each essay. The remaining three markers are linked to short YouTube videos related to the essay. The students' profile section on the other hand consists the largest number of markers amounting to 24 . These markers are linked to a 10 second self-recorded video of each student introducing themselves. The credit section which is the last page of the yearbook consists of one marker. The researcher labels this as a free marker since during the construction of the yearbook, even though the marker was positioned, there were no specific overlay for it. It is created as a backup marker should there be any need for additional overlay after printing. This comes handy as during the students' course completion ceremony, the students have come up with a farewell montage. This montage is not in the original plan. The idea for the montage crops up after the yearbook is printed. Such new idea is able to be accommodated into the yearbook by utilizing this free marker.

The third consideration is on the instrumentation. The integration of $\mathrm{AR}$ into reading materials requires both a software and a hardware. The software chosen for AR integration in the yearbook is the Aurasma application. It is an open source application and is among the popular applications used for AR in the education field. Aurasma is used by both the developer and the users of the yearbook. It can only be used online therefore internet connectivity is very important.

The hardware needed to access the integration of AR in the yearbook is the scanner. The scanner is important for both the developer and users of the yearbook. For the developer, the scanner is used in the process of tagging the overlays onto the markers while for the yearbook users, it is used to retrieve the overlays from the markers. The scanner must be equipped with a camera that is able to capture still images, record audios and videos, and equipped with a speaker, a microphone, a display screen and internet connection. Given these descriptions, smartphone is the most suitable scanner to be used for this yearbook. It does not only fit all the descriptions but it is also easily accessible since almost everyone nowadays owns one.

An instruction page on how to fully utilize the AR function of the yearbook is placed on page 6 of the yearbook. It is placed right after the front matters section and before the academic essay section since the integration of AR into the yearbook only starts after the academic essay section. It is important for readers to read the instruction before reading the academic essays to ensure a successful AR integration output. The instruction page includes a statement declaring the integration of AR in the year book. This is followed by a step by step instruction on how to acquire and utilize the 
tools needed to access AR features integrated in the yearbook, in this case the Aurasma application. Four illustrations consisting of pictures of the processes such as the selected tool (Aurasma), sample of the marker, scanning process, and result of the scan were also included in the instruction page to better assist readers in understanding the written instructions.

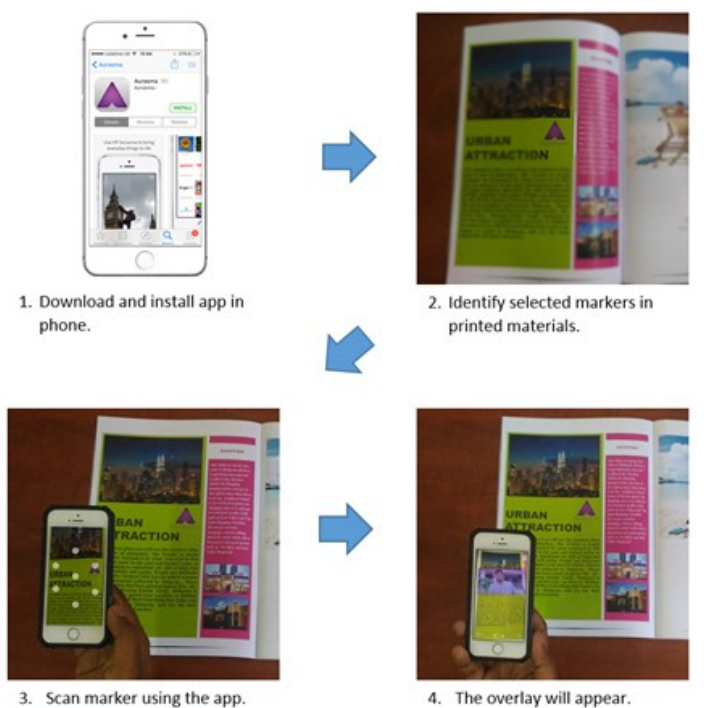

Fig. 2. Processes involved in retrieving AR content from the yearbook

Table 1. Reader appeal Mean and Standard Deviation for first and second reading

\begin{tabular}{lcccc}
\hline Item & \multicolumn{2}{c}{ First reading } & \multicolumn{2}{c}{ Second reading } \\
\cline { 2 - 5 } & Mean & SD & Mean & SD \\
Reader appeal & 4.1818 & 0.73855 & 4.4156 & 0.69492 \\
& & & & \\
\hline
\end{tabular}

With regard to the students' readership towards reading the AR yearbook, Table 1 presents the differences between reader appeal aspect for the first and second reading. The mean score for the first reading is 4.1818 ( $\mathrm{SD}=0.73855)$ while the mean score for the second reading is $4.4156(\mathrm{SD}=0.69492)$. Hence, the students in this study consider the AR integrated yearbook having more reader appeal rather than conventional reading material.

\section{Discussion}

This study suggests a few merits on integrating AR elements into the yearbook particularly on extending the period for more material inclusion, boosting the appeal of the book and increasing teamwork and collaboration in the project. On a more important note, this study has encouraged knowledge transfer in relation of AR to the students for further application in various learning contexts.
In addition, the integration of $\mathrm{AR}$ in the yearbook extends the period for more material inclusion. The development of the yearbook often ends upon printing; whereas AR accommodates to further development even years after the printing is completed. More content can be added even after the printing process, for example more videos, clips, audio, images and montage can be layered onto the finished product. In other words, the content of the yearbook can be updated, for example the student leader can add a new video of him graduating, travelling or getting married. This addition can be done even years after the book is published and given out to the graduating students. With this added value on being more interactive, dynamic and stimulating, the yearbook is revolutionized into a more meaningful reading material. AR gives room for further development even after the printing process has ended.

Besides, the use of AR also enhance the appeal of the yearbook. Colourful and visually rich books are often more attracting to be read. However, the printing cost may be an issue to the project developers. The integration of AR technology provide ways to make a yearbook more attractive and interactive without inducing too much cost. By using $\mathrm{AR}$, a college yearbook can be integrated with digital and multimedia content. The hardcopy of the conventional yearbook can be linked to additional digital content such as pictures, videos, clips, montages and others using AR. AR enhances a yearbook by making the pictures and articles on its page come to 'life'. Linking the articles to the multimedia and digital resources adds value to the publication by making it more interactive, explanatory and stimulating. The yearbook now can be enhanced and has value added since the interactive additional content can be integrated with the use of AR.

Furthermore, the use of AR in the yearbook also promotes teamwork and collaboration among students which otherwise are exclusive to the college editorial boards. The construction of the conventional yearbook involves only a small number of students who are the members of the editorial group. Often, the general readers are not involved in the development and only get to the see the end product. Therefore the yearbook is less interactive and may appear bland to some readers. The integration of AR into the yearbook is able to accommodate more involvement from a bigger number of students; teachers and even parents thus encourage more communal and collective effort. Moreover, with $\mathrm{AR}$, the yearbook is more interactive thus sustain more readerships.

To boot, the AR integration process described in this study can be further applied in various teaching and learning context. The prospect of the application in teaching and learning is vast. The teachers can apply the AR learned to enhance textbooks and teaching modules for example by linking videos for language and History subject, 3D simulations for biology subject and latest 
updates for exam answering techniques. The students can incorporate AR into their projects and reports. The use of yearbook in this study is just a medium to assist acceptance on the integration of AR technology towards the education setting which otherwise would be daunting. The process described is not only limited in revolutionizing yearbook but can also be utilized to enhance other conventional printed materials thus embracing the 21 st century teaching.

\section{Acknowledgments}

This study is supported by the Short Term Grant in part by Universiti Malaysia Perlis, under grant number 9001-00497.

\section{References:}

1. S.C. Yuen, G. Yaoyuneyong, \& E. Johnson, J.Edu. Tech.(JETDE) 4 ,1 (2011)

2. R. Azuma, Y. Baillot, R. Behringer, S. Feiner, S. Julier, \& B. MacIntyre, Comp. Grph. \& App IEEE, 21, 7 (2001)

3. H. Kaufmann, \& D. Schmalstieg, J. Comp. \& Grph, 27, 3 (2003)

4. M. Dunleavy, C. Dede, \& R. Mitchell, J. Sc. Edu. \& Tech 18, 1(2009)

5. M. Akcayir, Edu. Rsh \& Rev, 20, 1 (2017)

6. A. Dünser, L. Walker, H. Horner, \& D. Bentall, Proceedings of the 24th Australian ComputerHuman Interaction Conference on - OzCHI '12, 107-114. (2012)

7. Mahadzir, N. N., \& Phung, L. F. (2013). J. Rsch. \& Mthd. in Edu, 1, (2013)

8. Matcha, W., \& Rambli, D. R. A., Computer \& Information Science (ICCIS), 2012 International Conference on, 1123-1126. (2012)

9. Valarmathie, G., Zulkifli, A. N., Faisal Mohamed, N. F., Alwi, A., Saidin, A. Z., Mat, R. C., \& Abu Bakar, J. A. Proceedings of the 1st International Conference on Educational Studies (ICES2015). (2015)

10. Cheng, K. H. Aus. J. of Edu. Tech., 33,4, (2017) 